\title{
Terapia sostitutiva con glucocorticoidi somministrati per via sottocutanea in maniera pulsatile
}

\author{
Emanuela Arvat
}

Pubblicato online: 4 dicembre 2014

C Springer International Publishing AG 2014

Commento a:

\author{
Subcutaneous pulsatile glucocorticoid replacement \\ therapy. \\ G.M. Russell, C. Durant, A. Ataya, C. Papastathi, \\ R. Bhake, W. Woltersdorf, S. Lightman. \\ Clin Endocrinol (2014) 81:289-293
}

È noto che i pazienti affetti da insufficienza surrenalica, sia primitiva che secondaria, presentano una aumentata morbilità, con maggiore insorgenza di patologie metaboliche, osteoporosi, peggioramento della qualità di vita, e un'aumentata mortalità, soprattutto per patologie cardiovascolari. Un inadeguato trattamento sostitutivo glucocorticoide, in termini quantitativi e qualitativi, con un'alterata esposizione a elevati livelli circolanti giornalieri e una perdita del fisiologico ritmo circadiano delle concentrazioni di cortisolo sembra rivestire un ruolo importante nell'insorgenza delle complicanze di questa patologia.

Nello studio è stato sviluppato un metodo di somministrazione di cortisolo in maniera continua ad andamento pulsatile mediante pompa infusionale con cannula inserita a livello del tessuto sottocutaneo addominale.

Ventuno soggetti sani (7 femmine e 14 maschi) sono stati sottoposti all'esperimento in cui, dopo soppressione della surrenalica secrezione endogena mediante desametazone, è stata somministrata una dose di idrocortisone ogni 3 ore per
24 ore: $4 \mathrm{mg}$ alle ore $12.00,15.00 \mathrm{e} 18.00,2 \mathrm{mg}$ alle 21.00 , 24.00 e 03.00 e $0,5 \mathrm{mg}$ alle 06.00 e 09.00 , per un totale di dose giornaliera di 19,9 mg di idrocortisone. La cannula non ha indotto significativi effetti collaterali locali.

I dati hanno evidenziato che questa metodica permette di riprodurre il ritmo circadiano e la pulsatilità del cortisolo circolante.

Nel complesso, lo studio ha dimostrato che la somministrazione continua pulsatile con dosi scalari di idrocortisone mediante pompa infusionale è in grado di migliorare il profilo giornaliero delle concentrazioni di cortisolo, con un ritmo circadiano e un andamento pulsatile più simili a quelli fisiologici. Questa modalità di somministrazione sembra essere più fisiologica nel regolare le attività periferiche tissutali controllate dai glucocorticoidi circolanti, che presentano anch'esse un ritmo circadiano con una differente sensibilità agli effetti dei glucocorticoidi stessi. Infatti, non solo la circadianità della secrezione surrenalica ma anche la sua natura pulsatile sembrano essere cruciali per le risposte dei tessuti bersaglio e per il mantenimento delle funzioni biologiche dei tessuti sensibili ai glucocorticoidi. Non solo l'alterazione del ritmo circadiano ma anche la perdita della fisiologica pulsatilità della secrezione di cortisolo sembrano essere infatti associati ad alterazioni delle funzioni biologiche da esso controllate.
E. Arvat $(\varangle)$

Divisione di Endocrinologia Oncologica, Dipartimento di Scienze

Mediche, Università di Torino, Torino, Italia

e-mail: emanuela.arvat@unito.it 